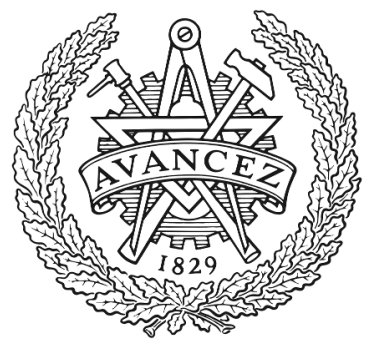

CHALMERS

UNIVERSITY OF TECHNOLOGY

\title{
Model-Based Machine Learning for Joint Digital Backpropagation and PMD Compensation
}

Downloaded from: https://research.chalmers.se, 2023-04-26 12:23 UTC

Citation for the original published paper (version of record):

Häger, C., Pfister, H., Bütler, R. et al (2020). Model-Based Machine Learning for Joint Digital

Backpropagation and PMD Compensation. Optical Fiber Communication Conference (OFC) 2020

N.B. When citing this work, cite the original published paper. 


\title{
Model-Based Machine Learning for Joint Digital Backpropagation and PMD Compensation
}

\author{
Christian Häger ${ }^{(1)}$, Henry D. Pfister ${ }^{(2)}$, Rick M. Bütler ${ }^{(3)}$, \\ Gabriele Liga $^{(3)}$, Alex Alvarado ${ }^{(3)}$ \\ ${ }^{(1)}$ Department of Electrical Engineering, Chalmers University, Gothenburg, Sweden \\ ${ }^{(2)}$ Department of Electrical and Computer Engineering, Duke University, Durham, USA \\ (3) Department of Electrical Engineering, Eindhoven University of Technology, Eindhoven, The Netherlands \\ christian.haeger@chalmers.se
}

\begin{abstract}
We propose a model-based machine-learning approach for polarizationmultiplexed systems by parameterizing the split-step method for the Manakov-PMD equation. This approach performs hardware-friendly DBP and distributed PMD compensation with performance close to the PMD-free case. (C) 2020 The Author(s)
\end{abstract}

OCIS codes: (060.0060) Fiber optics and optical communications, (060.2330) Fiber optics communications.

\section{Introduction}

The traditional application of machine learning to physical-layer communication replaces individual signal processing blocks (e.g., equalization or decoding) by neural networks (NNs) with the aim of learning betterperforming algorithms (or less complex ones) through data-driven optimization. More generally, one may regard the entire communication system design as an end-to-end reconstruction task and jointly optimize transmitter and receiver NNs [1]. Both traditional [2-4] and end-to-end learning [5-7] have received considerable attention for optical fiber systems. However, the reliance on NNs as universal (but sometimes poorly understood) function approximators makes it difficult to incorporate existing domain knowledge or interpret the obtained solutions.

Rather than relying on NNs, a different approach is to start from an existing model and parameterize it. For fiberoptic systems, this can be done for example by considering the split-step method (SSM) for numerically solving the nonlinear Schrödinger equation (NLSE). By viewing all chromatic-dispersion steps as general linear functions, one obtains a parameterized model similar to a multi-layer NN [8]. Compared to standard "black-box" models, this approach has several advantages: it leads to clear hyperparameter choices (such as the number of layers/steps); it provides good initializations for a gradient-based optimization; and it allows one to inspect the learned solutions in order to understand why they work well, thereby providing significant insight into the problem [8-10].

In this paper, we extend the model-based approach proposed in [8] to polarization-multiplexed (PM) systems by parameterizing the SSM for the Manakov-PMD equation. This leads to a multi-layer model alternating complexvalued $2 \times 2$ multiple-input multiple-output finite impulse response (MIMO-FIR) filters with nonlinear Kerr operators. The complexity of the filters can be reduced by decomposing them into separate filters for each polarization followed by memoryless rotation matrices [11]. As an application, we consider joint digital backpropagation (DBP) and polarization-mode dispersion (PMD) compensation, similar to [12-15]. Compared to previous work, the employed model uses hardware-friendly time-domain implementations $[16,17]$ and does not assume any knowledge about the particular PMD realizations along the link (i.e., differential group delays (DGDs) and polarization states), nor any knowledge about the total accumulated PMD. We show that our model converges reliably to a solution with performance close to the case where PMD is absent from the link.

\section{Supervised Machine Learning}

We start by reviewing the standard supervised learning setting for feed-forward NNs. A feed-forward NN with $M$ layers defines a mapping $\mathbf{y}=f_{\theta}(\mathbf{x})$ where the input vector $\mathbf{x} \in \mathscr{X}$ is mapped to the output vector $\mathbf{y} \in \mathscr{Y}$ by alternating between affine transformations $\mathbf{z}^{(i)}=\mathbf{W}^{(i)} \mathbf{x}^{(i-1)}+\mathbf{b}^{(i)}$ and pointwise nonlinearities $\mathbf{x}^{(i)}=\phi\left(\mathbf{z}^{(i)}\right)$ with $\mathbf{x}^{(0)}=\mathbf{x}$ and $\mathbf{x}^{(M)}=\mathbf{y}$. The parameter vector $\theta$ comprises all elements of the weight matrices $\mathbf{W}^{(1)}, \ldots, \mathbf{W}^{(M)}$ and vectors $\mathbf{b}^{(1)}, \ldots, \mathbf{b}^{(M)}$. Given a training set $S \subset \mathscr{X} \times \mathscr{Y}$ that contains a list of input-output pairs, training proceeds by minimizing the empirical loss $\mathscr{L}_{S}(\theta) \triangleq \frac{1}{|S|} \sum_{(\mathbf{x}, \tilde{\mathbf{y}}) \in S} \ell\left(f_{\theta}(\mathbf{x}), \tilde{\mathbf{y}}\right)$, where $\ell(\mathbf{y}, \tilde{\mathbf{y}})$ is the per-sample loss associated with returning the output $\mathbf{y}=f_{\theta}(\mathbf{x})$ when $\tilde{\mathbf{y}}$ is correct. When the training set is large, one typically optimizes $\theta$ using a variant of stochastic gradient descent (SGD). In particular, mini-batch SGD uses the parameter update $\theta_{t+1}=\theta_{t}-\alpha \nabla_{\theta} \mathscr{L}_{B_{t}}\left(\theta_{t}\right)$, where $\alpha$ is the step size and $B_{t} \subseteq S$ is the mini-batch used in the $t$-th step.

Supervised machine learning is not restricted to NNs and learning algorithms such as SGD can be applied to other function classes as well. In fact, prior to the current revolution in machine learning, communication engineers 

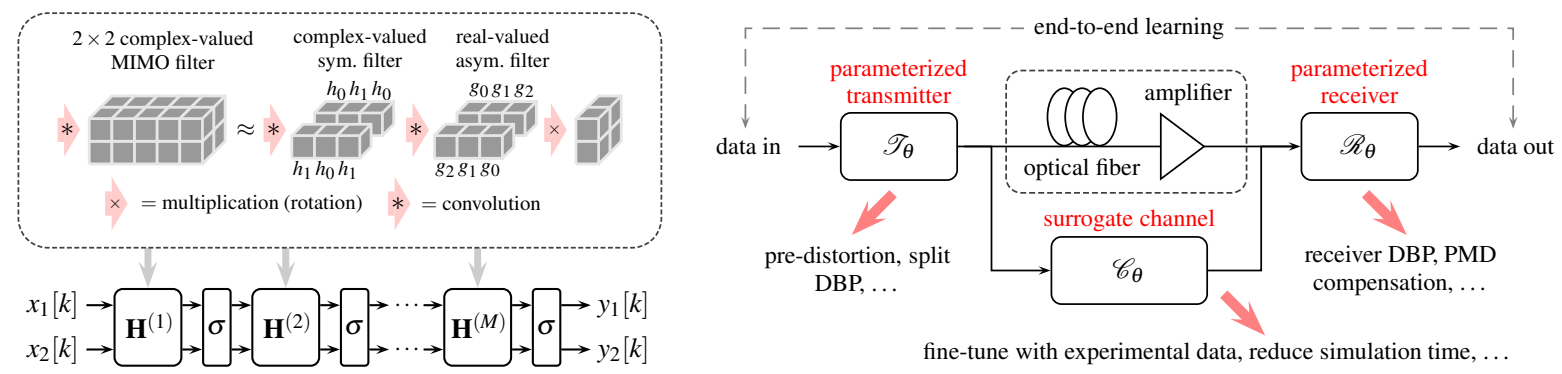

Fig. 1: Left: Block diagram of the proposed model $\mathbf{y}=f_{\theta}(\mathbf{x})$ based on the SSM for the Manakov-PMD equation, where all filters are free parameters $\theta=\left\{\mathbf{H}^{(1)}, \ldots, \mathbf{H}^{(M)}\right\}$ and $\sigma(\mathbf{x})=\mathbf{x} e^{j \gamma \frac{8}{9} \delta\|\mathbf{x}\|^{2}}$. The framed box illustrates the MIMO filter decomposition used for the numerical results. Right: Potential applications in the context of end-to-end learning [1], where $\mathscr{T}_{\theta}, \mathscr{R}_{\theta}, \mathscr{C}_{\theta}$ are parameterized functions.

were quite aware that system parameters (such as filter coefficients) could be learned using SGD. It was not at all clear, however, that more complicated parts of the system architecture could be learned as well. For example, in practice, PMD can be compensated by choosing the function $f_{\theta}$ as the convolution of the received signal with the impulse response of a complex-valued $2 \times 2$ MIMO-FIR filter, where $\theta$ are the filter coefficients. For a particular choice of the loss function $\ell$, applying SGD then maps into the well-known constant modulus algorithm [18].

\section{Model-Based Machine Learning for Polarization-Multiplexed Systems}

The evolution of PM signals over single-mode fiber can be described by a set of coupled NLSEs that takes into account the interactions between the two (degenerate) polarization modes. In birefringent fibers where polarization states change rapidly along the link, an appropriate approximation is given by the Manakov-PMD equation [19] $\frac{\partial}{\partial z} \mathbf{u}=\hat{\mathbf{D}} \mathbf{u}+j \gamma \frac{8}{9}\|\mathbf{u}\|^{2} \mathbf{u}$, where $\mathbf{u}=\mathbf{u}(t, z)=\left(u_{1}(t, z), u_{2}(t, z)\right)^{\top}$ is the Jones vector comprising the complex baseband signals in both polarizations, $\gamma$ is the nonlinear Kerr parameter, and $\hat{\mathbf{D}}$ is a linear operator that models attenuation, chromatic dispersion (CD), and PMD, see, e.g., [20, Eq. (1)].

Similar to the NLSE, the Manakov-PMD equation has no general closed-form solutions but can be solved numerically using the SSM. The SSM alternates linear and nonlinear steps, where the nonlinear steps are described by $\sigma(\mathbf{x})=\mathbf{x} e^{j \gamma \frac{8}{9} \delta\|\mathbf{x}\|^{2}}$ for $\mathbf{x} \in \mathbb{C}^{2}$ and step size $\delta$. The linear steps can be approximated by $2 \times 2$ MIMO-FIR filters in discrete time, which leads to the model shown in Fig. 1 (left). The proposed approach is to fully parameterize this model by regarding all filter coefficients in all steps as free parameters. In the context of end-to-end learning, as illustrated in Fig. 1 (right), such a model can serve for example as the basis for a fine-tuned channel model based on experimental data, using techniques similar to [21]. In this paper, we focus on receiver-side DBP, where the model can learn and apply arbitrary filter shapes to PM signals in a distributed fashion. The model can also be used at the transmitter to learn optimized pre-distortions [22,23] or split-DBP [24].

For multi-layer models, it is important to simplify the individual steps as much as possible to limit the overall complexity [11]. This is especially important since PMD is a time-varying impairment that requires adaptive filtering in practice. We therefore decompose each MIMO filter into three components as shown in Fig. 1 (left): (1) a complex-valued symmetric filter accounting for CD, (2) a real-valued asymmetric filter with "flipped" coefficients in different polarizations to approximate fractional-delay filters accounting for DGD, (3) a memoryless $2 \times 2$ rotation matrix. Such a decomposition considerably reduces the number of parameters and required hardware operations compared to a $2 \times 2 \mathrm{MIMO}$ filter. For the rotations, we assume that each matrix can be represented as $\left(\begin{array}{cc}a & -b^{*} \\ b & a^{*}\end{array}\right)$, where $a, b \in \mathbb{C}$, i.e., 4 real parameters per step. While this does not impose a unitary constraint on the rotation, we found that it leads to better SGD training compared to other parameterizations used in, e.g., [15].

\section{Numerical Results}

We consider the simulation setup in [13] with slightly adjusted parameters. In particular, we assume single-channel transmission of a 32 Gbaud PM signal (Gaussian symbols, root-raised cosine, roll-off 0.01 ) over $10 \times 100 \mathrm{~km}$ of fiber $\left(\alpha=0.2 \mathrm{~dB} / \mathrm{km}, \beta_{2}=-21.683 \mathrm{ps}^{2} / \mathrm{km}, \gamma=1.2 \mathrm{rad} / \mathrm{W} / \mathrm{km}\right.$ ), where optical amplifiers (noise figure $4.5 \mathrm{~dB}$ ) compensate for attenuation after each span. Forward propagation is simulated with 1000 uniform steps per span (StPS) and $192 \mathrm{GHz}$ simulation bandwidth. PMD was emulated at every step with a frequency-dependent Jones matrix $\mathbf{R}^{(i)} \mathbf{J}^{(i)}(\omega), i=1, \ldots, N=10000$, where $\mathbf{J}^{(i)}(\omega)=\operatorname{diag}\left(e^{-j \omega \frac{\tau_{i}}{2}}, e^{j \omega \frac{\tau_{i}}{2}}\right)$ is a first-order PMD matrix with DGD $\tau_{i}$ and $\mathbf{R}^{(i)}$ is a unitary rotation matrix. For generating a particular PMD realization $\left\{\tau_{i}, \mathbf{R}^{(i)}\right\}_{i=1}^{N}$ for the entire link, we use the same approach as described in [13] with PMD parameter $0.2 \mathrm{ps} / \sqrt{\mathrm{km}}$. At the receiver, the signal was low-pass filtered (64 GHz bandwidth) and downsampled to 2 samples/symbol for further processing. We started by training a 4-StPS model without rotations or DGD filters, assuming no PMD (i.e., $\tau_{i}=0, \mathbf{R}^{(i)}=\left(\begin{array}{ll}1 & 0 \\ 0 & 1\end{array}\right)$ 
for all $i$ ). The model has 41 steps based on the symmetric SSM, where the last step is a linear "half-step" without nonlinearity, followed by a matched filter (MF) and phase-offset correction. Mean-squared error loss is employed, which is essentially the same as standard learned DBP (LDBP) [8]. The solution is then used for two receiver setups accounting for PMD, where the corresponding performance serves as an upper bound, see Fig. 2 (blue squares; "ideal" DBP with 1000 StPS is also shown by the black circles). The first setup uses the same LDBP followed by the inverse of the PMD transfer function ma$\operatorname{trix} \mathbf{J}(\omega)=\prod_{i=1}^{N} \mathbf{R}^{(i)} \mathbf{J}^{(i)}(\omega)$, prior to the MF. No additional training is performed. Fig. 2 shows the mean effective SNR averaged over 40 PMD realizations (red triangles, shaded areas indicate standard deviation). As expected, PMD reduces the effectiveness of LDBP, which is similar to standard DBP. Next, the model was extended with rotations and 5-tap DGD filters. The CD filters are frozen to the LDBP solution during training, giving only 9 free parameters per step. The rotations are ran-

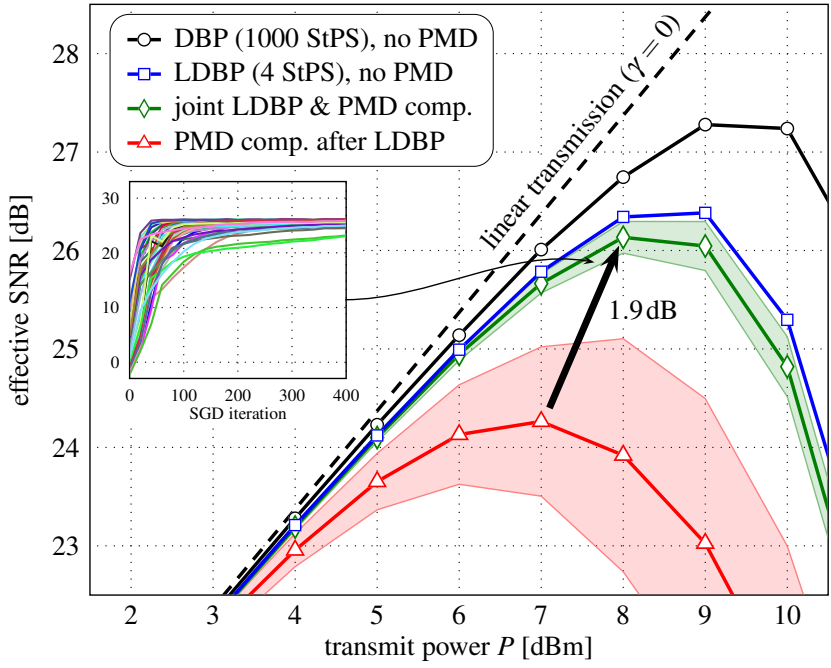

Fig. 2: Results for 40 PMD realizations (shaded areas are std. deviations). domly initialized and DGD filters are initialized to $(0,0,1,0,0)$. The model is trained for 1500 iterations with the Adam optimizer [25], learning rate 0.0005, and batch size 50. The results in Fig. 2 (green diamonds) show that the mean performance for the same 40 PMD realizations is improved by $1.9 \mathrm{~dB}$, which is only $0.2 \mathrm{~dB}$ away from LDBP without PMD. Moreover, the standard deviation is reduced by more than a factor of 6 . The training curves in Fig. 2 demonstrate a quick convergence behavior for all considered PMD realizations, where the starting SNR for the optimization is random due to the initialization of the rotations.

\section{Conclusions}

We have proposed a multi-layer machine-learning model for PM systems based on parameterizing the SSM for the Manakov-PMD equation. This model can be used for end-to-end learning as part of a parameterized transmitter or receiver implementation (or both), or as the basis for a fine-tuned channel model. In this paper, we have considered receiver-side DBP and PMD compensation, demonstrating performance close to the PMD-free case, without assuming any knowledge about the PMD realizations along the link or the total accumulated PMD.

Acknowledgements: This work is part of a project that has received funding from the European Union's Horizon 2020 research and innovation programme under the Marie Skłodowska-Curie grant agreement No. 749798. The work of H. D. Pfister was supported in part by the National Science Foundation (NSF) under Grant No. 1609327. The work of A. Alvarado and G. Liga has received funding from the European Research Council (ERC) under the European Union's Horizon 2020 research and innovation programme (grant agreement No. 757791). Any opinions, findings, recommendations, and conclusions expressed in this material are those of the authors and do not necessarily reflect the views of these sponsors.

\section{References}

1. T. O'Shea and J. Hoydis, "An introduction to deep learning for the physical layer," IEEE Trans. Cogn. Commun. Netw. 3, 563-575 (2017).

2. T. S. R. Shen and A. P. T. Lau, "Fiber nonlinearity compensation using extreme learning machine for DSP-based coherent communication systems," Proc. OECC (2011).

3. A. M. Jarajreh et al., "Artificial neural network nonlinear equalizer for coherent optical OFDM," IEEE Photon. Technol. Lett. 27, 387-390 (2015).

4. J. Estarán et al., "Artificial neural networks for linear and non-linear impairment mitigation in high-baudrate IM/DD systems," Proc. ECOC (2016).

5. S. Li et al., "Achievable information rates for nonlinear fiber communication via end-to-end autoencoder learning," Proc. ECOC (2018).

6. R. T. Jones et al., "Deep learning of geometric constellation shaping including fiber nonlinearities," Proc. ECOC (2018)

7. B. Karanov et al., "End-to-end deep learning of optical fiber communications," J. Light. Technol. 36, 4843-4855 (2018).

8. C. Häger and H. D. Pfister, "Nonlinear interference mitigation via deep neural networks," Proc. OFC (2018).

9. C. Häger and H. D. Pfister, "Deep learning of the nonlinear Schrödinger equation in fiber-optic communications," Proc. ISIT (2018).

10. M. Lian et al., "What can machine learning teach us about communications?" Proc. ITW (2018).

11. C. Häger et al., "Revisiting multi-step nonlinearity compensation with machine learning," Proc. ECOC (2019).

12. K. Goroshko et al., "Overcoming performance limitations of digital back propagation due to polarization mode dispersion," Proc. ICTON (2016).

13. C. B. Czegledi et al., "Polarization-mode dispersion aware digital backpropagation," Proc. ECOC (2016).

14. C. B. Czegledi et al., "Digital backpropagation accounting for polarization-mode dispersion," Opt. Express 25, 1903-1915 (2017).

15. G. Liga et al., "A PMD-adaptive DBP receiver based on SNR optimization," Proc. OFC (2018).

16. C. Fougstedt et al., "Time-domain digital back propagation: Algorithm and finite-precision implementation aspects," Proc. OFC (2017).

17. C. Fougstedt et al., "ASIC implementation of time-domain digital backpropagation with deep-learned chromatic dispersion filters," Proc. ECOC (2018).

18. S. J. Savory, "Digital filters for coherent optical receivers," Opt. Express 16, 804-817 (2008).

19. P. K. A. Wai et al., "Stability of solitons in randomly varying birefringent fibers," Opt. Lett. 16, 1231 (1991).

20. E. Ip, “Nonlinear compensation using backpropagation for polarization-multiplexed transmission," J. Light. Technol. 28, 939-951 (2010).

21. T. J. O'Shea et al., "Approximating the void: Learning stochastic channel models from observation with variational generative adversarial networks," Proc. ICNC (2019).

22. R.-J. Essiambre and P. J. Winzer, "Fibre nonlinearities in electronically pre-distorted transmission,” Proc. ECOC (2005)

23. K. Roberts et al., "Electronic precompensation of optical nonlinearity," IEEE Photon. Technol. Lett. 18, $403-405$ (2006).

24. D. Lavery et al., "The benefit of split nonlinearity compensation for single-channel optical fiber communications," IEEE PTL 28, 1803-1806 (2016).

25. D. P. Kingma and J. Ba, "Adam: A method for stochastic optimization,", Proc. ICLR (2014). 